PROCEEDINGS OF THE

AMERICAN MATHEMATICAL SOCIETY

Volume 137, Number 11, November 2009, Pages 3797-3806

S 0002-9939(09)09964-X

Article electronically published on July 10, 2009

\title{
NON-REAL EIGENVALUES OF SINGULAR INDEFINITE STURM-LIOUVILLE OPERATORS
}

\author{
JUSSI BEHRNDT, QUTAIBEH KATATBEH, AND CARSTEN TRUNK
}

(Communicated by Chuu-Lian Terng)

\begin{abstract}
We study a Sturm-Liouville expression with indefinite weight of the form $\operatorname{sgn}\left(-d^{2} / d x^{2}+V\right)$ on $\mathbb{R}$ and the non-real eigenvalues of an associated selfadjoint operator in a Krein space. For real-valued potentials $V$ with a certain behaviour at $\pm \infty$ we prove that there are no real eigenvalues and that the number of non-real eigenvalues (counting multiplicities) coincides with the number of negative eigenvalues of the selfadjoint operator associated to $-d^{2} / d x^{2}+V$ in $L^{2}(\mathbb{R})$. The general results are illustrated with examples.
\end{abstract}

\section{INTRODUCTION}

We consider a singular Sturm-Liouville differential expression of the form

$$
\operatorname{sgn}(x)\left(-f^{\prime \prime}(x)+V(x) f(x)\right), \quad x \in \mathbb{R},
$$

with the signum function as indefinite weight and a real-valued locally summable function $V$. Under the assumption that $-d^{2} / d x^{2}+V$ is in the limit point case at $+\infty$ and $-\infty$ the maximal operator $A$ associated to (1.1) is selfadjoint in the Krein space $\left(L^{2}(\mathbb{R}),[\cdot, \cdot]\right)$, where the indefinite inner product $[\cdot, \cdot]$ is defined by

$$
[f, g]=\int_{\mathbb{R}} f(x) \overline{g(x)} \operatorname{sgn}(x) d x, \quad f, g \in L^{2}(\mathbb{R}) .
$$

The spectral properties of indefinite Sturm-Liouville operators differ essentially from the spectral properties of selfadjoint Sturm-Liouville operators in the Hilbert space $L^{2}(\mathbb{R})$; e.g. the real spectrum of $A$ necessarily accumulates to $+\infty$ and $-\infty$ and $A$ may have non-real eigenvalues which possibly accumulate to the real axis (see [3, 4, 9, 14, 16, 20]). For further indefinite Sturm-Liouville problems, applications and references, see, e.g., [2, 6, 7, 11, 13, 15, 23, 26].

The main objective of this paper is to study the number of non-real eigenvalues of the operator $A$. For this it will be assumed that the negative spectrum of the selfadjoint definite Sturm-Liouville operator $B f=-f^{\prime \prime}+V f$ consists of $\kappa<\infty$ eigenvalues. Then the hermitian form $[A \cdot, \cdot]$ has exactly $\kappa$ negative squares, and it follows from the considerations in [9] and 21] that the spectrum $\sigma(A)$ of $A$ in the open upper half-plane $\mathbb{C}^{+}$consists of at most $\kappa$ eigenvalues (counting multiplicities). Inspired by results of I. Knowles from [18, 19, we give a sufficient condition on $V$ such that $\sigma(A) \cap \mathbb{C}^{+}$consists of exactly $\kappa$ eigenvalues (counting multiplicities) and

Received by the editors November 14, 2008, and, in revised form, February 14, 2009, and February 23, 2009.

2000 Mathematics Subject Classification. Primary 47A10; Secondary 47B50.

(C)2009 American Mathematical Society Reverts to public domain 28 years from publication 
the continuous spectrum of $A$ covers the whole real line; see Theorem 2.3 and Corollary 2.4 below. These results can be viewed as a partial answer to the open problem $\mathrm{X}$ in [26, p. 300]. We present two explicitly solvable examples illustrating our results. In the first example, potentials of hyperbolic secant type are considered and with the help of numerical methods we find $\kappa$ different eigenvalues in $\mathbb{C}^{+}$. The second example shows that, in general, non-real eigenvalues of $A$ may have nontrivial Jordan chains, and hence the number of distinct eigenvalues in $\mathbb{C}^{+}$is less than $\kappa$.

\section{Eigenvalues of indefinite Sturm-Liouville operators}

In this section we consider the indefinite Sturm-Liouville differential expression on $\mathbb{R}$ given by (1.1), where $V: \mathbb{R} \rightarrow \mathbb{R}$ is a real function with $V \in L_{\mathrm{loc}}^{1}(\mathbb{R})$. We equip the Hilbert space $\left(L^{2}(\mathbb{R}),(\cdot, \cdot)\right)$ with the indefinite inner product $[\cdot, \cdot \cdot]$ defined in $(1.2)$ and denote the corresponding Krein space $\left(L^{2}(\mathbb{R}),[\cdot, \cdot]\right)$ by $L_{\mathrm{sgn}}^{2}(\mathbb{R})$. As a corresponding fundamental symmetry we choose $J:=\operatorname{sgn}(\cdot)$; hence we have $[\cdot, \cdot]=(J \cdot, \cdot)$ and $[J \cdot, \cdot]=(\cdot, \cdot)$. For the basic properties of indefinite inner product spaces and linear operators therein, we refer to [1] and [8].

Suppose that the definite Sturm-Liouville differential expression

$$
-\frac{d^{2}}{d x^{2}}+V
$$

is in the limit point case at $+\infty$ and $-\infty$; that is, for each $\lambda \in \mathbb{C} \backslash \mathbb{R}$ there exist (up to scalar multiples) unique solutions of the differential equation $-y^{\prime \prime}+V y=\lambda y$ which are square integrable in a neighbourhood of $+\infty$ and $-\infty$, respectively. A sufficient criterion for (2.1) to be in the limit point case at $\pm \infty$ is, e.g.

$$
\liminf _{|x| \rightarrow \infty} \frac{V(x)}{x^{2}}>-\infty
$$

cf. [25, Satz 13.27] or [26, Example 7.4.1]11 Denote by $\mathcal{D}_{\max }$ the linear space of all $f \in L^{2}(\mathbb{R})$ such that $f$ and $f^{\prime}$ are absolutely continuous and $-f^{\prime \prime}+V f \in L^{2}(\mathbb{R})$ holds. Then it is well-known that the maximal operator

$$
B f:=-f^{\prime \prime}+V f, \quad \operatorname{dom} B=\mathcal{D}_{\max },
$$

associated to (2.1) is selfadjoint in the Hilbert space $L^{2}(\mathbb{R})$ and all eigenvalues are real and simple; i.e., $\operatorname{dim} \operatorname{ker}(B-\lambda)=1$ for $\lambda \in \sigma_{p}(B)$. As a consequence we obtain the following statement for the operator $A=J B$.

Proposition 2.1. Assume that (2.1) is in the limit point case at $\pm \infty$. Then the indefinite Sturm-Liouville operator defined by

$$
(A f)(x)=\operatorname{sgn}(x)\left(-f^{\prime \prime}(x)+V(x) f(x)\right), \quad x \in \mathbb{R}, \quad \operatorname{dom} A=\mathcal{D}_{\max },
$$

is selfadjoint in the Krein space $L_{\mathrm{sgn}}^{2}(\mathbb{R})$, and the eigenspaces $\operatorname{ker}(A-\lambda), \lambda \in \sigma_{p}(A)$, have dimension one.

In the following it will be assumed that condition (I), stated below, holds.

(I) The set $\sigma(B) \cap(-\infty, 0)$ consists of $\kappa<\infty$ eigenvalues.

\footnotetext{
${ }^{1}$ In the formulation of [26] Example 7.4.1] a minus sign is missing.
} 
Hence, the selfadjoint operator $B$ in the Hilbert space $L^{2}(\mathbb{R})$ is semi-bounded from below and the eigenvalues do not accumulate to zero from the negative half-axis. A sufficient condition for (I) to hold is, e.g., $\int_{\mathbb{R}}\left(1+x^{2}\right)|V(x)| d x<\infty$ for continuous $V$; cf. 22.

We collect some properties of the non-real spectrum of the indefinite SturmLiouville operator $A$ in the next proposition. Recall first that the spectrum of a selfadjoint operator in a Krein space is symmetric with respect to the real axis and denote by $\mathcal{L}_{\lambda}(A)$ the algebraic eigenspace of $A$ corresponding to an eigenvalue $\lambda$.

Proposition 2.2. The spectrum of the indefinite Sturm-Liouville operator $A$ in the open upper half-plane $\mathbb{C}^{+}$consists of at most finitely many eigenvalues with

$$
\sum_{\lambda \in \sigma_{p}(A) \cap \mathbb{C}^{+}} \operatorname{dim} \mathcal{L}_{\lambda}(A) \leq \kappa
$$

where $\kappa$ is as in (I). In particular, for some $l \leq \kappa$ we have

$$
\mathbb{C} \backslash \mathbb{R} \subset \rho(A) \cup\left\{\lambda_{1}, \bar{\lambda}_{1}, \ldots, \lambda_{l}, \bar{\lambda}_{l}\right\} .
$$

If $V(x)=V(-x), x \in \mathbb{R}$, then $\sigma_{p}(A)$ is symmetric with respect to the imaginary axis.

Proof. Assumption (I) and the relation $[A f, f]=(J A f, f)=(B f, f), f \in \mathcal{D}_{\max }$, imply that the hermitian form $[A \cdot, \cdot]$ has exactly $\kappa$ negative squares; that is, there exists a $\kappa$-dimensional subspace $\mathcal{M}$ in $\mathcal{D}_{\max }$ such that $[A f, f]<0$ if $f \in \mathcal{M}, f \neq 0$, but no $(\kappa+1)$-dimensional subspace with this property. This, together with wellknown properties of operators with $\kappa$ negative squares (see, e.g., 21, [9] and [5, Theorem 3.1 and $\S 4.2]$ ) imply (2.4) and (2.5).

Moreover, if $V$ is symmetric, then $\lambda$ is an eigenvalue of $A$ with corresponding eigenfunction $x \mapsto y(x)$ if and only if $-\lambda$ is an eigenvalue of $A$ with corresponding eigenfunction $x \mapsto y(-x)$. Therefore, as $\sigma_{p}(A)$ is symmetric with respect to the real axis, $\sigma_{p}(A)$ is also symmetric with respect to the imaginary axis.

Under some additional assumptions on $V$ we prove in Theorem 2.3 the absence of eigenvalues on the real axis and, hence, improve the estimate in (2.4). We mention that in [15, Section 4] a similar result is proved if $V$ satisfies $\int_{\mathbb{R}}(1+|x|)|V(x)| d x<\infty$. By $\sigma_{c}(A)$ we denote the continuous part of the spectrum of $A$, i.e. the set of all $\lambda \in \sigma(A) \backslash \sigma_{p}(A)$ such that the range of $A-\lambda$ is dense.

Theorem 2.3. Assume that condition (I) holds and that there exist real functions $q$ and $r$ with $V=q+r$ such that $\lim _{|x| \rightarrow \infty} r(x)=\lim _{|x| \rightarrow \infty} q(x)=0, r$ is locally of bounded variation and

$$
\lim _{t \rightarrow \infty} \frac{1}{\log t} \int_{-t}^{t}|q(x)| d x=\lim _{t \rightarrow \infty} \frac{1}{\log t} \int_{-t}^{t}|d r(x)|=0,
$$

where $d r$ denotes the measure induced by $r$. Then $\sigma_{c}(A) \backslash\{0\}=\mathbb{R} \backslash\{0\}$ and hence zero is the only possible real eigenvalue of the indefinite Sturm-Liouville operator $A$. If, in addition, $0 \notin \sigma_{p}(B)$, then we have $\sigma_{c}(A)=\mathbb{R}$ and

$$
\sum_{\lambda \in \sigma_{p}(A) \cap \mathbb{C}^{+}} \operatorname{dim} \mathcal{L}_{\lambda}(A)=\kappa
$$


Proof. Let $\lambda$ be an eigenvalue of $A$ and let $y$ be a corresponding eigenfunction. Then $y$ satisfies the equations

$$
-y^{\prime \prime}(x)+V(x) y(x)=\lambda y(x), \quad x \in(0, \infty),
$$

and

$$
y^{\prime \prime}(x)-V(x) y(x)=\lambda y(x), \quad x \in(-\infty, 0) .
$$

Condition (2.6) implies

$$
\lim _{t \rightarrow \infty} \frac{1}{\log t} \int_{0}^{t}|q(x)| d x=\lim _{t \rightarrow \infty} \frac{1}{\log t} \int_{0}^{t}|d r(x)|=0 .
$$

This and [19, Theorem 3.2] applied to (2.8) yield $\lambda \notin(0, \infty)$. Similarly, (2.6) implies

$$
\lim _{t \rightarrow \infty} \frac{1}{\log t} \int_{-t}^{0}|q(x)| d x=\lim _{t \rightarrow \infty} \frac{1}{\log t} \int_{-t}^{0}|d r(x)|=0,
$$

and, with [19, Theorem 3.2] applied to (2.9), we find $\lambda \notin(-\infty, 0)$. Therefore, as a selfadjoint operator in a Krein space has no real points in the residual spectrum (see, e.g., 8, Corollary VI.6.2]), we obtain

$$
(\sigma(A) \cap(\mathbb{R} \backslash\{0\})) \subset \sigma_{c}(A) \text { and } \sigma_{p}(A) \subset\{0\} \cup \mathbb{C} \backslash \mathbb{R} .
$$

Moreover, from $A=J B$ we get $0 \in \sigma_{p}(A)$ if and only if $0 \in \sigma_{p}(B)$. Hence, if $0 \notin \sigma_{p}(B)$, we conclude $\sigma_{p}(A) \subset \mathbb{C} \backslash \mathbb{R}$. Since the operator $A$ has exactly $\kappa$ negative squares (cf. the proof of Proposition 2.2), it follows from, e.g. [5, Theorem 3.1], that $A$ has $\kappa$ eigenvalues (counted with multiplicities) in $\mathbb{C}^{+}$and thus (2.7) holds.

It remains to show $\mathbb{R} \subset \sigma(A)$. For this, consider the differential expressions $\ell_{+}=-\frac{d^{2}}{d x^{2}}+V$ on $\mathbb{R}^{+}$and $\ell_{-}=\frac{d^{2}}{d x^{2}}-V$ on $\mathbb{R}^{-}$. By assumption $\ell_{+}$and $\ell_{-}$are regular at zero and in the limit point case at $\infty$ and $-\infty$, respectively. Let $A_{+}$ and $A_{-}$be selfadjoint realizations of $\ell_{+}$and $\ell_{-}$in the Hilbert spaces $L^{2}\left(\mathbb{R}^{+}\right)$and $L^{2}\left(\mathbb{R}^{-}\right)$, respectively, e.g. corresponding to Dirichlet boundary conditions at zero. Under our assumptions

$$
\lim _{x \rightarrow \infty} V(x)=0 \text { and } \lim _{x \rightarrow-\infty} V(x)=0,
$$

and it is well known that $[0, \infty) \subset \sigma\left(A_{+}\right)$and $(-\infty, 0] \subset \sigma\left(A_{-}\right)$hold. Since the rank of the operator

$$
(A-\lambda)^{-1}-\left(\left(A_{+} \times A_{-}\right)-\lambda\right)^{-1}, \quad \lambda \in \rho(A) \cap \rho\left(A_{+} \times A_{-}\right),
$$

is at most two and $\sigma\left(A_{+} \times A_{-}\right)=\mathbb{R}$, we conclude $\mathbb{R} \subset \sigma(A)$.

A sufficient condition on $V$ such that condition (I), (2.6) and $0 \notin \sigma_{p}(B)$ hold is given in the next corollary; cf. [24, Theorem 14.10], [26, §6.3] and [18, Remark after Corollary 3.3].

Corollary 2.4. Assume that there exists $x_{0}>0$ with

$$
-\frac{1}{4 x^{2}} \leq V(x) \leq \frac{3}{4 x^{2}} \quad \text { for all } \quad x \in \mathbb{R} \backslash\left(-x_{0}, x_{0}\right) .
$$

Then $\sigma_{c}(A)=\mathbb{R}$ and

$$
\sum_{\lambda \in \sigma_{p}(A) \cap \mathbb{C}^{+}} \operatorname{dim} \mathcal{L}_{\lambda}(A)=\kappa
$$


Remark 2.5. We mention that (even under the condition (2.10) ) for $\lambda \in \sigma_{p}(A)$, $\operatorname{dim} \mathcal{L}_{\lambda}(A)>1$ may happen; i.e. there exists a Jordan chain of length greater than one, and the non-real spectrum does not consist of $\kappa$ distinct eigenvalues. In Section 4 we give an example for an indefinite singular Sturm-Liouville operator with such a Jordan chain corresponding to a non-real eigenvalue.

\section{A numerical EXAmple: Hyperbolic SeCANT potentials}

In this section we compute the non-real eigenvalues of singular indefinite SturmLiouville operators with potentials given by

$$
V_{\kappa}(x)=-\kappa(\kappa+1) \operatorname{sech}^{2}(x), \quad x \in \mathbb{R} \text { and } \kappa \in \mathbb{N},
$$

with the help of the software package Mathematica (Wolfram Research).

It is well known (see, e.g., [12]) that the number of negative eigenvalues of the definite Sturm-Liouville operator $B f=-f^{\prime \prime}+V_{\kappa} f$ in (2.2) is exactly $\kappa$ and condition (I) from Section 2 holds. Moreover, $V_{\kappa}$ satisfies (2.10), and hence by Theorem 2.3 and Corollary 2.4 the continuous spectrum of the indefinite SturmLiouville operator

$$
(A f)(x)=\operatorname{sgn}(x)\left(-f^{\prime \prime}(x)+V_{\kappa}(x) f(x)\right), \quad x \in \mathbb{R}, \quad \operatorname{dom} A=\mathcal{D}_{\max },
$$

in the Krein space $L_{\mathrm{sgn}}^{2}(\mathbb{R})$ coincides with $\mathbb{R}$ and $\sum_{\lambda \in \sigma_{p}(A) \cap \mathbb{C}^{+}} \operatorname{dim} \mathcal{L}_{\lambda}(A)=\kappa$ holds. In order to determine the non-real eigenvalues of $A$, we divide the problem into two parts,

$$
\begin{aligned}
-y^{\prime \prime}(x ; \lambda)+V_{\kappa}(x) y(x ; \lambda) & =\lambda y(x ; \lambda), & & x \in \mathbb{R}^{+}, \\
y^{\prime \prime}(x ; \lambda)-V_{\kappa}(x) y(x ; \lambda) & =\lambda y(x ; \lambda), & & x \in \mathbb{R}^{-} .
\end{aligned}
$$

Since the potential $V_{\kappa}$ in (3.1) satisfies $V_{\kappa}(x)=V_{\kappa}(-x)$ for $x \in \mathbb{R}$, it follows that a function $x \mapsto h(x ; \lambda), x \in \mathbb{R}^{+}$, is a so-

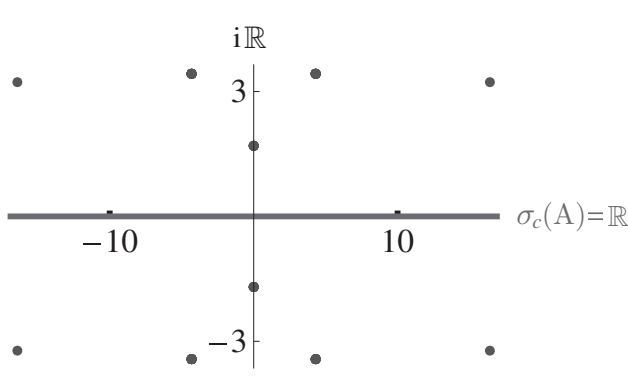

Figure 1 . The case $\kappa=5$. lution of the first differential equation if and only if $x \mapsto h(-x ;-\lambda), x \in \mathbb{R}^{-}$, is a solution of the second differential equation in (3.2). Moreover, as both singular endpoints $\infty$ and $-\infty$ are in the limit point case, each of the equations in (3.2) has (up to scalar multiples) a unique square integrable solution. Since the functions in $\operatorname{dom} A$ and their derivatives are continuous at the point 0 it follows that $\lambda \in \mathbb{C} \backslash \mathbb{R}$ is an eigenvalue of $A$ if and only if for the square integrable solution $x \mapsto h(x ; \lambda), x \in \mathbb{R}^{+}$, of the first equation in (3.2),

$$
h(0 ; \lambda)=\gamma h(0 ;-\lambda) \quad \text { and } \quad h^{\prime}(0 ; \lambda)=-\gamma h^{\prime}(0 ;-\lambda)
$$

hold for some $\gamma \in \mathbb{C}$. For non-real $\lambda$ we have $h(0 ; \lambda) \neq 0$ and $h(0 ;-\lambda) \neq 0$, and therefore (3.3) is satisfied if and only if the function

$$
\mu \mapsto M(\mu):=\frac{h^{\prime}(0 ; \mu)}{h(0 ; \mu)}+\frac{h^{\prime}(0 ;-\mu)}{h(0 ;-\mu)}, \quad \mu \in \mathbb{C} \backslash \mathbb{R},
$$




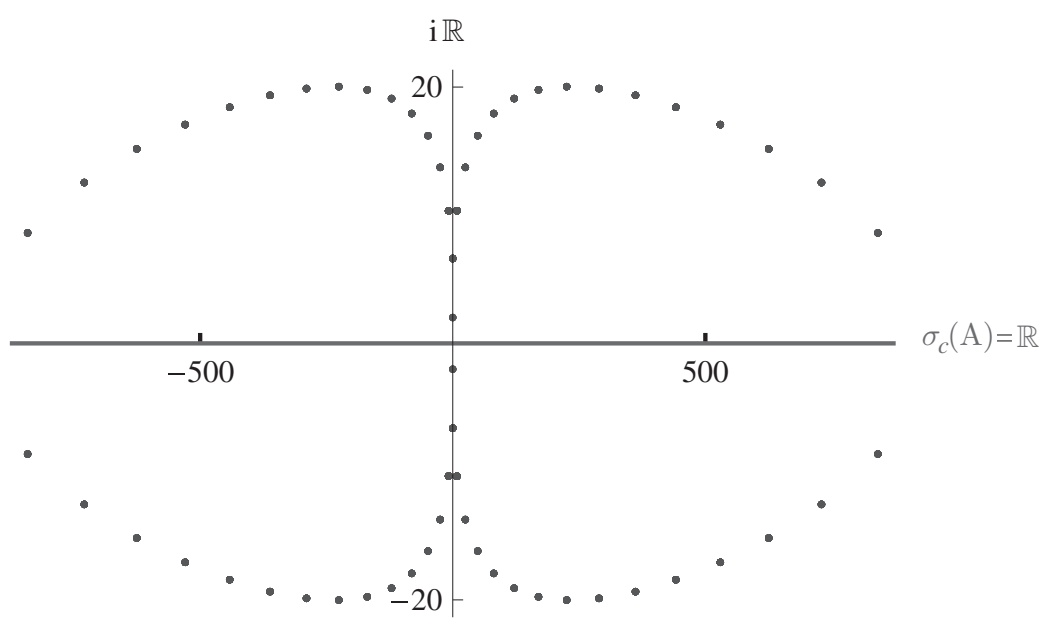

Figure 2. The operator $(A y)(x):=\operatorname{sgn}(x)\left(-y^{\prime \prime}(x)+V_{30}(x) y(x)\right)$, $x \in \mathbb{R}$, where $V_{30}(x)=-30 \cdot 31 \operatorname{sech}^{2}(x)$ has $\kappa=30$ pairs of nonreal eigenvalues.

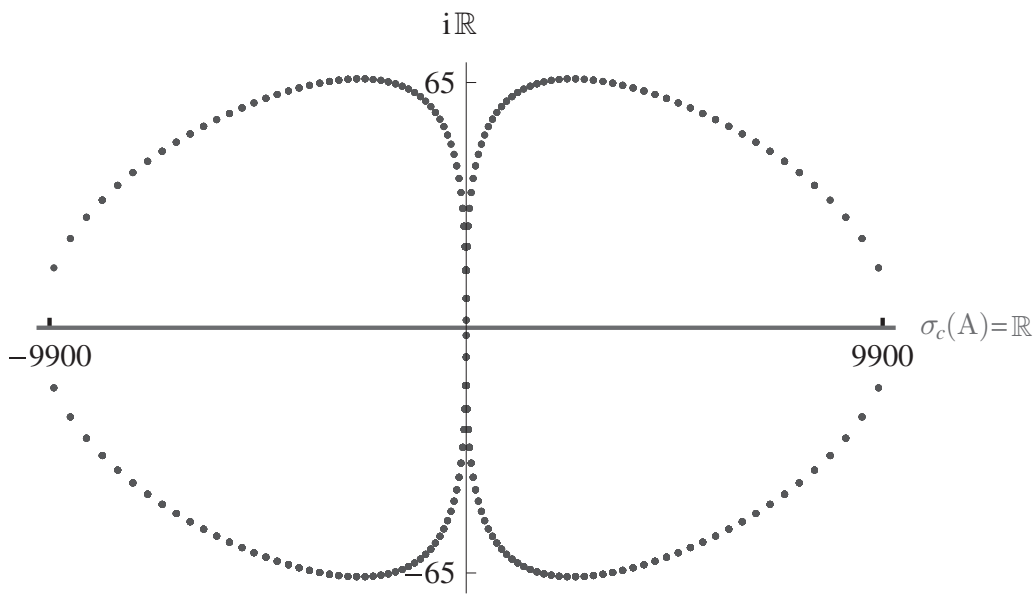

Figure 3. The operator $(A y)(x):=\operatorname{sgn}(x)\left(-y^{\prime \prime}(x)+\right.$ $\left.V_{100}(x) y(x)\right), x \in \mathbb{R}$, where $V_{100}(x)=-100 \cdot 101 \operatorname{sech}^{2}(x)$ has $\kappa=100$ pairs of non-real eigenvalues.

has a zero at $\lambda$. As the equations in (3.2) are explicitly solvable in terms of Legendre functions we can determine numerically the zeros of $M$ within the working default precision of the software package Mathematica.

Figures 1, 2 and 3 show the non-real eigenvalues of $A$ for the cases $\kappa=5, \kappa=30$ and $\kappa=100$. Here we find 5,30 and 100 , respectively, distinct eigenvalues in $\mathbb{C}^{+}$, and hence $\operatorname{dim} \mathcal{L}_{\lambda}(A)=1$ for each eigenvalue $\lambda \in \mathbb{C} \backslash \mathbb{R}$; cf. Remark 2.5. Note also that by the symmetry of $V_{\kappa}$ there is at least one pair of eigenvalues on the imaginary axis if $\kappa$ is odd. 


\section{A Counterexample: Jordan Chains of Singular IndeFinite STURM-LiOUVILLE OPERATORS}

In this section we show that the geometric eigenspaces of a singular indefinite Sturm-Liouville operator in $L_{\mathrm{sgn}}^{2}(\mathbb{R})$ in general do not coincide with the algebraic eigenspaces. In other words, there exist eigenvalues with non-trivial Jordan chains, and hence the number of non-real distinct eigenvalues is in general smaller than the dimension of the algebraic eigenspace corresponding to the non-real spectrum; cf. Remark 2.5. An explicit example of a non-trivial Jordan chain of a regular indefinite Sturm-Liouville operator can be found in [10].

We consider a family

$$
\left(A_{\eta} f\right)(x)=\operatorname{sgn}(x)\left(-f^{\prime \prime}(x)+V_{\eta}(x) f(x)\right), \quad x \in \mathbb{R}, \quad \operatorname{dom} A_{\eta}=\mathcal{D}_{\max }, \quad \eta \geq 0,
$$

of indefinite Sturm-Liouville operators in the Krein space $L_{\text {sgn }}^{2}(\mathbb{R})$, where the potentials $V_{\eta}, \eta \geq 0$, are given by

$$
V_{\eta}(x)=\left\{\begin{array}{ll}
0 & |x| \geq 1, \\
-\eta & |x|<1,
\end{array} \quad \eta \geq 0 .\right.
$$

The operators $A_{\eta}, \eta \geq 0$, are selfadjoint in $L_{\mathrm{sgn}}^{2}(\mathbb{R})$, and according to Theorem 2.3 and Corollary 2.4 there are no real eigenvalues and $\sigma_{c}\left(A_{\eta}\right)$ covers the whole real line. In the sequel we will show that the following statement holds.

Proposition 4.1. There exist an $\eta_{0}>0$ and a $\lambda_{0} \in \mathbb{C}^{+}$such that

$$
2=\operatorname{dim} \operatorname{ker}\left(A_{\eta_{0}}-\lambda_{0}\right)^{2}>\operatorname{dim} \operatorname{ker}\left(A_{\eta_{0}}-\lambda_{0}\right)=1 .
$$

In order to determine the eigenvalues of the operators $A_{\eta}$, we first consider the underlying differential equations (3.2) with $V_{\kappa}$ replaced by $V_{\eta}$. The same reasoning as in Section 3 shows that the non-real eigenvalues of $A_{\eta}$ are given by the zeros of the function

$$
\lambda \mapsto M_{\eta}(\lambda):=\frac{h_{\eta}^{\prime}(0 ; \lambda)}{h_{\eta}(0 ; \lambda)}+\frac{h_{\eta}^{\prime}(0 ;-\lambda)}{h_{\eta}(0 ;-\lambda)}, \quad \lambda \in \mathbb{C} \backslash \mathbb{R},
$$

where $h_{\eta}(\cdot ; \lambda)$ is the square integrable solution of $-y^{\prime \prime}+V_{\eta} y=\lambda y$ on $\mathbb{R}^{+}$. Denote by $\sqrt{\cdot}$ the branch of the square root with cut along $[0, \infty)$ and $\sqrt{x} \geq 0$ for $x \in[0, \infty)$. Then it is easy to check that for $\lambda \notin[0, \infty)$ the function

$$
h_{\eta}(x ; \lambda)= \begin{cases}\exp (i \sqrt{\lambda} x) & x>1, \\ \alpha_{\eta}(\lambda) \exp (i \sqrt{\lambda+\eta} x)+\beta_{\eta}(\lambda) \exp (-i \sqrt{\lambda+\eta} x) & x \in[0,1]\end{cases}
$$

where

$$
\alpha_{\eta}(\lambda)=\frac{1}{2}\left(1+\sqrt{\lambda(\lambda+\eta)^{-1}}\right) \exp (i(\sqrt{\lambda}-\sqrt{\lambda+\eta}))
$$

and

$$
\beta_{\eta}(\lambda)=\frac{1}{2}\left(1-\sqrt{\lambda\left(\lambda+\eta^{-1}\right.}\right) \exp (i(\sqrt{\lambda}+\sqrt{\lambda+\eta}))
$$

and its multiples are square integrable solutions of the first equation in (3.2) with $V_{\kappa}$ replaced by $V_{\eta}$.

The function $M_{\eta}$ in (4.1) can be expressed in terms of $\alpha_{\eta}$ and $\beta_{\eta}$ in the following form:

$$
M_{\eta}(\lambda)=i \sqrt{\lambda+\eta} \frac{\alpha_{\eta}(\lambda)-\beta_{\eta}(\lambda)}{\alpha_{\eta}(\lambda)+\beta_{\eta}(\lambda)}+i \sqrt{\eta-\lambda} \frac{\alpha_{\eta}(-\lambda)-\beta_{\eta}(-\lambda)}{\alpha_{\eta}(-\lambda)+\beta_{\eta}(-\lambda)} .
$$



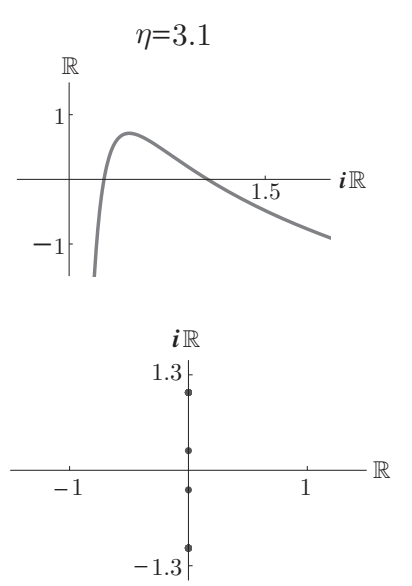
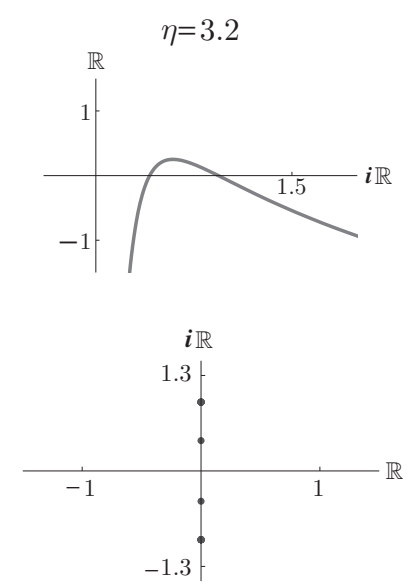
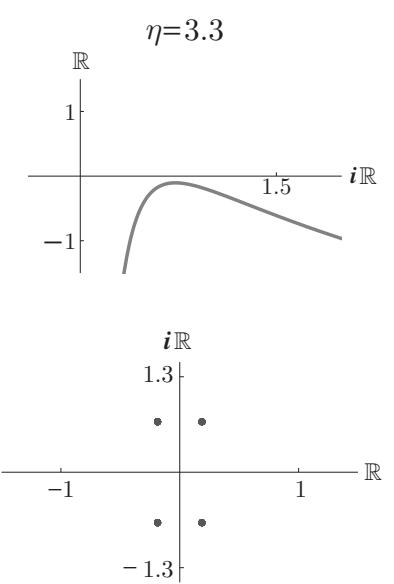

FiguRE 4 . In the first row the function $\mu \mapsto M_{\eta}(i \mu)$ is plotted for $\mu>0$ and $\eta=3.1, \eta=3.2$ and $\eta=3.3$, respectively. In the second row the corresponding non-real eigenvalues of the operators $A_{3.1}$, $A_{3.2}$ and $A_{3.3}$ are shown.

We note that the values $M_{\eta}(i \mu), \mu \in \mathbb{R} \backslash\{0\}$, are real since the solutions fulfill $h_{\eta}(x, \bar{\lambda})=\overline{h_{\eta}(x, \lambda)}$ for $\lambda \in \mathbb{C} \backslash \mathbb{R}$. Let us summarize some observations in the following lemma.

Lemma 4.2. A non-real number $\lambda$ is an eigenvalue of the indefinite Sturm-Liouville operator $A_{\eta}$ if and only if $M_{\eta}(\lambda)=0$. The restriction of $M_{\eta}$ onto the imaginary axis is a real-valued function, and the non-real eigenvalues of $A_{\eta}$ are symmetric with respect to the real and imaginary axes.

One can check numerically that the selfadjoint operator $B_{\eta}=-\frac{d^{2}}{d x^{2}}+V_{\eta}$, $\operatorname{dom} B_{\eta}=\mathcal{D}_{\max }$, in the Hilbert space $L^{2}(\mathbb{R})$ has exactly two negative eigenvalues for $\eta=3.1, \eta=3.2$ and $\eta=3.3$; cf. [12. By Corollary 2.4 for these $\eta$ the spectral subspace of $A_{\eta}$ correponding to the eigenvalues in the upper half-plane $\mathbb{C}^{+}$ has dimension two.

The plots in the first row of Figure 4 show the function $\mu \mapsto M_{\eta}(i \mu), \mu \in \mathbb{R}^{+}$, for $\eta=3.1, \eta=3.2$ and $\eta=3.3$, respectively. For $\eta=3.1$ and $\eta=3.2$ the two zeros are the eigenvalues of $A_{3.1}$ and $A_{3.2}$ in the upper half-plane $\mathbb{C}^{+}$which lie on the positive imaginary axis. These eigenvalues and their counterparts in $\mathbb{C}^{-}$are plotted in the second row of Figure 4. For $\eta=3.3$ the function $\mu \mapsto$ $M_{\eta}(i \mu)$ has no zeros on the positive imaginary axis. Recall that a finite system of eigenvalues is continuous under perturbations small in norm; see [17, IV.3.5]. Hence the continuity and symmetry of the eigenvalues of $A_{\eta}$ imply that the eigenvalues of $A_{3.3}$ are located as in the right lower plot in Figure 4. This can also be checked numerically by computing the non-real roots of $M_{3.3}$; see also Table 1, Again by continuity properties of the point spectrum there exists an $\eta_{0} \in(3.2,3.3)$ such that the spectrum of $A_{\eta_{0}}$ in $\mathbb{C}^{+}$(and hence also in $\mathbb{C}^{-}$) consists only of one eigenvalue $\lambda_{0}$ on the imaginary axis with corresponding algebraic eigenspace of dimension two. Recall that the dimension of the geometric eigenspaces of $A_{\eta_{0}}$ is at most one since $\infty$ and $-\infty$ are in the limit point case. Hence there exists a Jordan chain of length 
TABLE 1. In bold face is the (approximative) value of $\eta$ where the eigenvalues $\lambda_{1, \eta}$ and $\lambda_{2, \eta}$ of $A_{\eta}$ in $\mathbb{C}^{+}$coincide and we have a Jordan chain of length two. With further increasing $\eta$, the eigenvalues $\lambda_{1, \eta}$ and $\lambda_{2, \eta}$ move away from the imaginary axis.

\begin{tabular}{|c|c|c|}
\hline$\eta$ & $\lambda_{1, \eta}$ & $\lambda_{2, \eta}$ \\
\hline 3.10000000000 & $0.26723799239 i$ & $1.05923928894 i$ \\
\hline 3.26656565972 & $0.64287403712 i$ & $0.72260288819 i$ \\
\hline 3.26796097363 & $0.67270918484 i$ & $0.69312432044 i$ \\
\hline 3.26805876683 & $0.68293354062 i$ & $0.68293354054 i$ \\
\hline $\mathbf{3 . 2 6 8 0 5 8 7 6 6 8 5}$ & $\mathbf{0 . 6 8 2 9 2 9 2 8 8 5 6} i$ & $\mathbf{0 . 6 8 2 9 2 9 2 8 8 5 6} i$ \\
\hline 3.26805890000 & $0.0003766+0.6829292 i$ & $-0.0003766+0.6829292 i$ \\
\hline 3.27021280983 & $0.0479471+0.6832050 i$ & $-0.0479471+0.6832050 i$ \\
\hline 3.28021280983 & $0.1143198+0.6844929 i$ & $-0.1143198+0.6844929 i$ \\
\hline 3.30000000000 & $0.1866925+0.687078 i$ & $-0.1866925+0.687078 i$ \\
\hline
\end{tabular}

two of $A_{\eta_{0}}$ at the eigenvalue $\lambda_{0}$ (and $\bar{\lambda}_{0}$ ). We remark that for the function (4.1) we have $M_{\eta_{0}}\left(\lambda_{0}\right)=M_{\eta_{0}}^{\prime}\left(\lambda_{0}\right)=0$.

\section{ACKNowledgement}

The authors thank Richard L. Hall for helpful discussions.

\section{REFERENCES}

1. T.Ya. Azizov and I.S. Iokhvidov, Linear Operators in Spaces with an Indefinite Metric, John Wiley and Sons, Chichester, New York, 1989. MR1033489 (90j:47042)

2. R. Beals, Indefinite Sturm-Liouville problems and half-range completeness, J. Differential Equations 56 (1985), 391-407. MR780497 (86i:34032)

3. J. Behrndt, On the spectral theory of singular indefinite Sturm-Liouville operators, J. Math. Anal. Appl. 334 (2007), 1439-1449. MR2338672 (2008e:47104)

4. J. Behrndt, Q. Katatbeh and C. Trunk, Accumulation of complex eigenvalues of indefinite Sturm-Liouville operators, J. Phys. A: Math. Theor. 41 (2008), 244003. MR2455801

5. J. Behrndt and C. Trunk, On the negative squares of indefinite Sturm-Liouville operators, J. Differential Equations 238 (2007), 491-519. MR2341434 (2008j:47035)

6. P. Binding and P. Browne, Eigencurves for two-parameter selfadjoint ordinary differential equations of even order, J. Differential Equations 79 (1989), 289-303. MR.1000691 (90e:47038)

7. P. Binding and H. Volkmer, Eigencurves for two-parameter Sturm-Liouville equations, SIAM Review 38 (1996), 27-48. MR1379040 (97f:34015)

8. J. Bognar, Indefinite Inner Product Spaces, Springer, Berlin, 1974. MR0467261 (57:7125)

9. B. Curgus and H. Langer, A Kreı̆n space approach to symmetric ordinary differential operators with an indefinite weight function, J. Differential Equations 79 (1989), 31-61. MR997608 (90j:47060)

10. J. Fleckinger and A.B. Mingarelli, On the eigenvalues of nondefinite elliptic operators, Differential equations, Proc. Conf., Birmingham, Ala., 1983, North-Holland Math. Stud. 92, North-Holland, Amsterdam, 1984, 219-227. MR799351 (86j:35072)

11. W. Greenberg, C.V.M. van der Mee and V. Protopopescu, Boundary Value Problems in Abstract Kinetic Theory, Oper. Theory Adv. Appl. 23, Birkhäuser Verlag, Basel, 1987. MR896904 (88k:82156)

12. R.L. Hall, Square-well representation for potentials in quantum mechanics, J. Math. Phys. 33 (1992), 3472-3476. MR 1182920 (93g:81018)

13. H.G. Kaper, C.G. Lekkerkerker and J. Hejtmanek, Spectral Methods in Linear Transport Theory, Oper. Theory Adv. Appl. 5, Birkhäuser Verlag, Basel, 1982. MR685594 (85i:82079) 
14. I.M. Karabash and M.M. Malamud, Indefinite Sturm-Liouville operators $(\operatorname{sgn} x)\left(-\frac{d^{2}}{d x^{2}}+\right.$ $q(x))$ with finite-zone potentials, Operators and Matrices 1 (2007), 301-368. MR.2344680 (2008g:47083)

15. I.M. Karabash, A.S. Kostenko and M.M. Malamud, The similarity problem for J-nonnegative Sturm-Liouville operators, J. Differential Equations 246 (2009), 964-997. MR2474582

16. I.M. Karabash and C. Trunk, Spectral properties of singular Sturm-Liouville operators with indefinite weight $\operatorname{sgn} x$, to appear in Proc. Roy. Soc. Edinburgh Sect. A.

17. T. Kato, Perturbation Theory for Linear Operators, Grundlehren der Mathematischen Wissenschaften 132, Springer-Verlag, Berlin-New York, 1976. MR0407617 (53:11389)

18. I. Knowles, On the number of $L^{2}$-solutions of second order linear differential equations, Proc. Roy. Soc. Edinburgh Sect. A 80 (1978), 1-13. MR529564 (80a:34017)

19. I. Knowles, On the location of eigenvalues of second order linear differential operators, Proc. Roy. Soc. Edinburgh Sect. A 80 (1978), 15-22. MR.529565 (80d:34026)

20. Q. Kong, M. Möller, H. Wu and A. Zettl, Indefinite Sturm-Liouville problems, Proc. Roy. Soc. Edinburgh Sect. A 133 (2003), no. 3, 639-652. MR.1983691(2004f:34039)

21. H. Langer, Spectral functions of definitizable operators in Krĕ̌n spaces, Proceedings of a Functional Analysis conference held at Dubrovnik, Yugoslavia, November 2-14, 1981, Lecture Notes in Mathematics 948, Springer-Verlag, Berlin-Heidelberg-New York, 1982, 1-46. MR672791 (84g:47034)

22. B.M. Levitan and I.S. Sargsjan, Sturm-Liouville and Dirac Operators, Kluwer, Dordrecht, 1991. MR:1136037 (92i:34119)

23. H. Volkmer, Sturm-Liouville problems with indefinite weights and Everitt's inequality, Proc. Roy. Soc. Edinburgh Sect. A 126 (1996), 1097-1112. MR1415825 (98a:34096)

24. J. Weidmann, Spectral Theory of Ordinary Differential Operators, Lecture Notes in Math. 1258, Springer-Verlag, Berlin, 1987. MR.923320 (89b:47070)

25. J. Weidmann, Lineare Operatoren in Hilberträumen, Teil II, Teubner, Stuttgart, 2003. MR 2382320 (2008k:47002)

26. A. Zettl, Sturm-Liouville Theory, Math. Surveys Monogr. 121, Amer. Math. Soc., Providence, RI, 2005. MR2170950 (2007a:34005)

Department of Mathematics MA 6-4, Technische Universität Berlin, Strasse des 17. Juni 136, D-10623 Berlin, Germany

E-mail address: behrndt@math.tu-berlin.de

Department of Mathematics and Statistics, Faculty of Science and Arts, Jordan University of Science And Technology, P.O. Box 3030, Irbid 22110, Jordan

E-mail address: qutaibeh@yahoo.com

Department of Mathematics, Technische Universität Ilmenau, Postfach 100565, D-98684 Ilmenau, Germany

E-mail address: carsten.trunk@tu-ilmenau.de 